Kamran E. Jafarzade

DOI: 10.25045/jpit.v09.i1.10

Institute of Information Technology of ANAS, Baku, Azerbaijan

kamran@science.az

\title{
COMPARATIVE ANALYSIS OF THE SOFTWARE USED IN SUPERCOMPUTER TECHNOLOGIES
}

The article considers the classification of the types of supercomputer architectures, such as MPP, SMP and cluster, including software and application programming interfaces: MPI and PVM. It also offers a comparative analysis of software in the study of the dynamics of the distribution of operating systems $(O S)$ for the last year of use in supercomputer technologies. In addition, the effectiveness of the use of CentOS software on the scientific network "AzScienceNet" is analyzed.

Keywords: supercomputer, operating system, software, cluster, SMP-architecture, MPP-architecture, MPI, PVM, CentOS.

\section{Introduction}

Supercomputer is a computer with high computing performance compared to a regular computer. Supercomputers are often used for scientific and engineering applications that need to process very large databases or perform a large number of calculations. The performance of a supercomputer is measured in floating-point operations per second (FLOPS) instead of millions of instructions per second (MIPS). Since 2015, the supercomputers performing up to quadrillion FLOPS have started to be developed. Modern supercomputers represent a large number of high performance server computers, which are interconnected via a local high-speed backbone to achieve the highest performance [1].

Supercomputers were originally introduced in the 1960s and bearing the name or monogram of the companies such as Seymour Cray of Control Data Corporation (CDC), Cray Research over the next decades. By the end of the 20th century, massively parallel supercomputers with tens of thousands of available processors started to be manufactured.

The supercomputers are often used to work with the applications that require more complex calculations. This feature distinguishes the supercomputers from the servers and mainframes - high performance computers designed to solve only typical tasks. Exclusively, supercomputer software are only the specialized software for monitoring and control of the certain types of computers, including unique software environments created in the computing centers as "own" and unique configurations of supercomputer systems [2].

\section{Supercomputer architecture}

The following types of architectures are widely used in the development of supercomputers: MPP (massively parallel processing), SMP (symmetric multiprocessing) and cluster.

SMP is a symmetric multiprocessor architecture. An important feature of the system with SMP architecture is the physical memory shared by all processors. Memory serves, in particular, for distributing messages among the processors. Furthermore, all computing devices have the same rights and the same addressing for all existing memory cells when accessing it. Therefore, SMPis called symmetric. SMP includes a multiprocessor hardware and software architecture, in which two or more identical processors connected to the same shared memory. These processors have full access to all input/output devices, and are controlled by an operating system that correspondingly handles all processors. The whole system runs under a single OS (usually UNIXlike, however for Intel platforms it is supported by Windows NT) [3].

Currently, SMP-architecture is not as relevant as MPP and cluster, and is almost not used in currently available supercomputers. The share of supercomputer architectures: MPP and cluster, according to the statistics obtained from the top500.org for the end of the first half of 2017, is provided in Figure 1 [4]. 


\section{Architecture System Share}

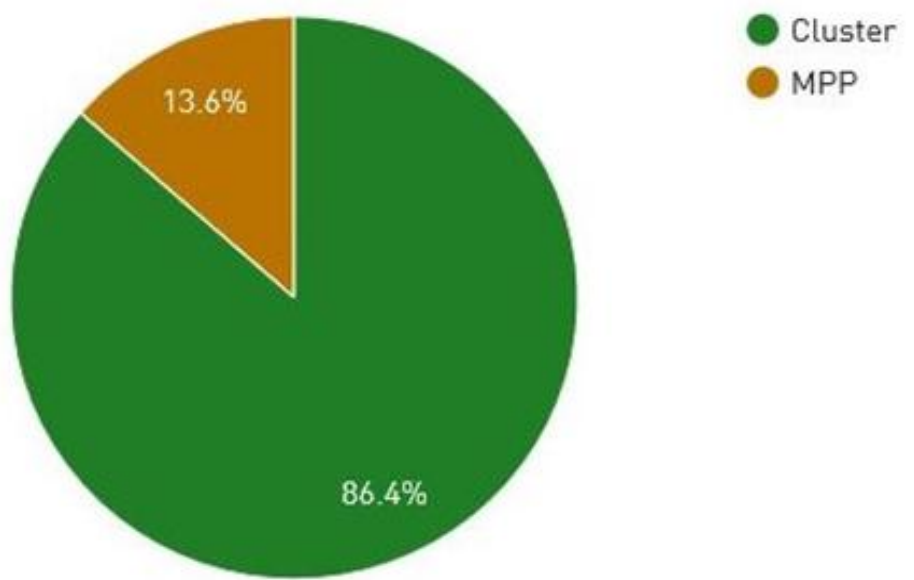

Fig.1. The share of supercomputer architectures: MPP and cluster

MPP is a massively parallel architecture. The main feature of this architecture is that the memory is physically divided. In this case, the system is built from separate modules containing a processor, a local bank of operating memory, two communication routers or a network adapter, sometimes, hard drives and/or other input/output devices.

There are two types of performance of the operating system on MPP-architecture machines. First, a full-fledged operating system runs exclusively on the controlling machine with each operating module that runs a minimal version of the operating system and ensures the work of the branch of the parallel application located in it. Second, each module has a full UNIX-like system installed separately on each module.

Cluster is a group of servers and other resources operating as a single system and providing high performance and in some cases, load balancing and parallel processing [5].

There are the following main types of cluster:

- Fail-over cluster (High-availability cluster);

- Load balancing cluster;

- Computing cluster;

- Grid systems [6].

The clusters often use single-processor computers, two- or four-processor SMP-servers as the computing nodes. Each node operates under its own copy of the operating system, which is mostly used by standard operating systems: Linux, NT, Solaris, etc.

\section{Organization of parallel computing}

The opportunity of distributing the computing load and organizing the data transfer among the processors is of great importance for the organization of parallel computing.

The most popular supercomputer software, including parallel or distributed computer systems, are the application programming interfaces based on MPI (Message Passing Interface) and PVM (Parallel Virtual Machine) and software-based solution that allows creating supercomputers even on the basis of ordinary workstations and PC. The most common programs among GNU/Linux users are as follows:

- distcc, MPICH (Message Passing Interface CHameleon) - specialized tools for parallelizing the program's performance.

- Linux Virtual Server, Linux-HA - nodal software for distributing requests among computing servers.

- MOSIX, openMosix, Kerrighed, OpenSSI (Single-System Image) - full-featured clustered environments built into the core, which automatically distribute the tasks among homogeneous nodes that create a single system environment among nodes. 
MPI is a message-passing interface that is a standardized and portable messaging system. The standard defines the syntax and semantics of the core of the library sub-program, which are useful for a wide range of users who write portable message passing programs in $\mathrm{C}, \mathrm{C}++$ and Fortran [7]. The goal of MPI is high performance, scalability and portability. MPI remains as the dominant model used in modern high-performance computing. There are several proven and effective implementations of MPI, many of which are open or public. Special MPI implementations are built and continue to be created for different OS and data transmission networks used in the clusters. MPICH package is a free MPI implementation.

PVM (parallel virtual machine) is a software package that enables the use of a set of heterogeneous computers connected to the local network, which operates under the Unix operating system as a single large parallel computer. PVM complex provides the operation of the parallel programs in computing environments. SPMD (Single Program Multiple Data) and MPMD (Multiple Programs Multiple Data) types of applications can be built in the system. It is compatible with the applications written in different programming languages and accurately converts the data when transmission through the nodes of different architectures. PVM is an open and freely distributed system that allows users to use existing hardware to perform rather complex tasks at a minimal cost. Actually, PVM is the current standard for distributed computing $[8,9]$.

\section{Operating systems of supercomputer}

Linux system is very popular among the supercomputers with $64.2 \%$ of the total for the end of the first half of 2017, according to statistics provided by top500.org, as shown in Figure 2 [4]. Next come CentOS and Cray Linux Environment with a relatively smaller share of use, $12.6 \%$ and 8.6\%, respectively. In addition, the commercial distribution of Linux - SUSE Linux Enterprise Server, founded by SUSE, which is intended for corporate use, still continues to be used. Relatively earlier Microsoft Windows Compute Cluster Server (CCS) 2008 is an integrated platform for various high-performance computing. With the use of this platform, various calculations are performed on clusters with 100 and even 1000 nodes [10].
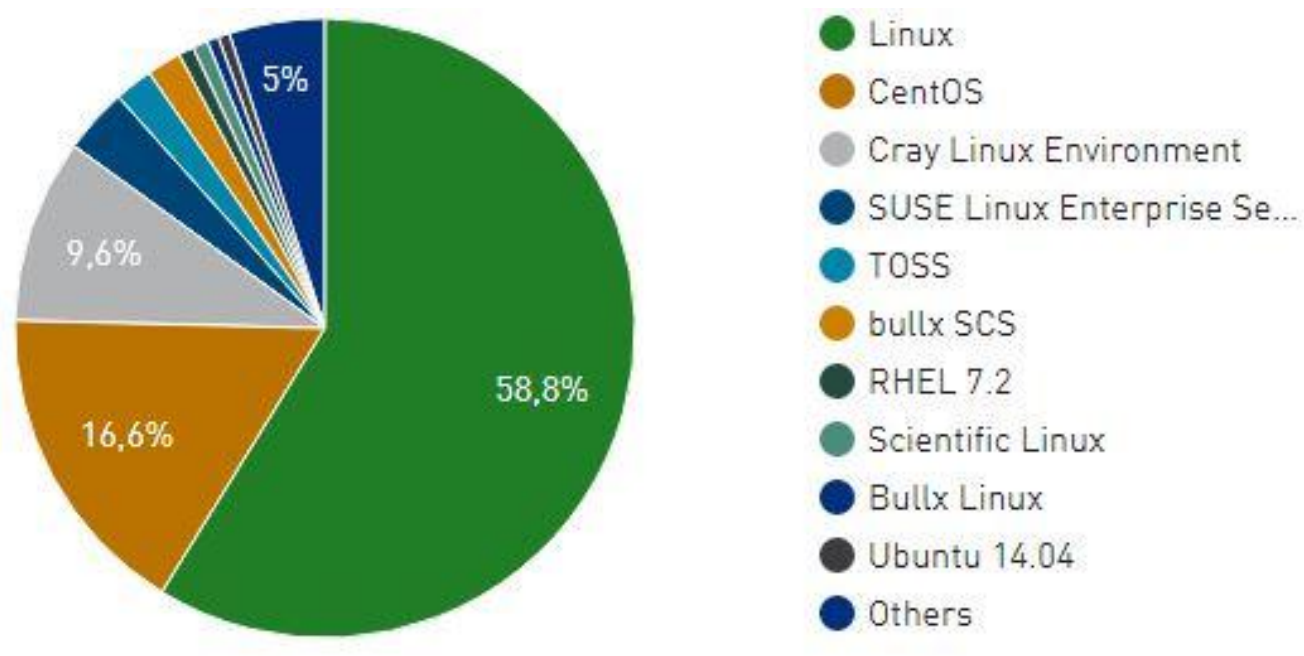

Fig.2. Share of the use of operating systems in supercomputers

Linux. The Linux operating system is often used in the versions particularly optimized for distributed parallel computing. The Linux system supports SMP-architecture. Moreover, its implementation does not require critical changes in the core; in the framework of the gradual development of the core, a minimal set of necessary primitives is added to it. For absolutely independent processes, that do not use shared resources, SMP support is not required: they will 
work using each of own processors with their own amount of RAM if possible, and their overall performance in a dual processor configuration will be almost two times higher than in a uniprocessor configuration. The use of exceptional coordination tools is necessary only if two processes attempt simultaneously to access the same resource often located in memory. In this case, the operating system must organize the access that one processor does not accidentally damage the results of the other. To this end, the Linux OS provides a system for controlling the access to memory areas and blocks up writing and reading [11].

Unicos (all the letters are officially uppercase - UNICOS) is the name of different versions of the Unix operating system manufactured by Cray for own supercomputers. Unicos is the following operating system of the company after the release of the Cray Operating System (COS). That provided the operation of network clusters and compatibility at the source code level with some other Unix versions. Unicos was first introduced as the operating system of Cray-2 supercomputer, and later, was transferred to other Cray models. Cray released several different operating systems entitled Unicos. The original version of Cray Unix, based on Unix System V, was used in supercomputers Cray-1, Cray-2, X-MP, Y-MP, and C90. Since the release of version 2.1, UNICOS/lc it has officially been called the Cray Linux Environment (CLE).

Windows Compute Cluster Server 2008. The first pilot product in the field of supercomputer technologies produced by the Microsoft was the development of Windows Server Compute Cluster Edition 2003 (CCE) and Windows Compute Cluster Server 2003 (WCCS) designed to meet the requirements of a wide range of HPC (High Performance Computing) applications. WCCS differs from CCE only for the presence of Microsoft Compute Cluster Pack (CCP). Nevertheless, due to the lack of functionality of WCCS in Microsoft, an operating system was created for cluster systems based on its latest development - Server 2008. This was the release of the HPC Server 2008.

Both CCE and WCCS systems are only released as x64 edition, i.e. x86 edition of these products are not available. Hardware requirements for CCE and WCCS are similar to the requirements for the x64 edition of Windows Server 2003 Standard. To ensure the highperformance hardware (x64 architecture), these systems are additionally supported by RDMA (Remote Direct Memory Access) for high-speed connections.

Computing clusters have a number of basic requirements that are fulfilled by installing the Compute Cluster Pack - a stand - alone installation package that includes the following components:

- Built-in task scheduler;

- MPI interface support for MPICH2 standard;

- User tools and tools for managing cluster resources [12].

\section{Main tasks of the CentOS operating system used in "AzScienceNet"}

CentOS (Community Enterprise Operating System) is a completely free distributive of the operating system, based on the Linux core, released by RedHat, May 2004. It is completely obtained from the distributive of Red Hat Enterprise Linux (RHEL). CentOS provides a free enterprise-class computing platform and aims to support 100 percent binary compatibility with Red Hat source code.

CentOS is one of the most popular Linux distributives in the hosting industry. Due to the binary compatibility of CentOS with RHEL, it is reasonably compatible with most Linux programs. Most hosting control panels use CentOS as the preferred Linux distributive.

In addition, CentOS was used as the default OS in the Amazon Cluster Compute Instance (CCI) project, the essence of which is to rent the power of a large number of standard rack servers.

CentOS OS has the following advantages:

- High-level security. Recently, CentOS is used for the given server on an increasing number of machines. A part of possible omissions is eliminated even before the release of its update. 
- High performance. Like most Linux-like systems, CentOS provides high-speed performance, which is an indispensable factor in the operation of large projects.

- Completely free. Although Red Hat has a paid support too, nonetheless, CentOS is still totally free of charge.

- Convenient GUI is not just a user-friendly interface, but also a convenient tool for performing the necessary actions. CentOS is fully compatible with popular GUI packages GNOME and KDE.

- The function of building Live $\mathrm{CD}$, which helps to get the system edition with the parameters for the needs of a particular project.

- Easy update and search for applications with the YUM (Yellow Dog Updater, Modified) module, which makes it easy to find the necessary files, applications, update packets that can be automated.

- Access to the server hardware, which provides more precise settings that can optimize performance [13].

The file system CentOS has a similar hierarchical structure as the Linux system, it is organized in the form of a tree with one original top called the root directory. The root directory is the parent directory for the entire Linux file system. The slash "/" is used to denote the root directory. Fig.3 illustrates the hierarchy of this file system.

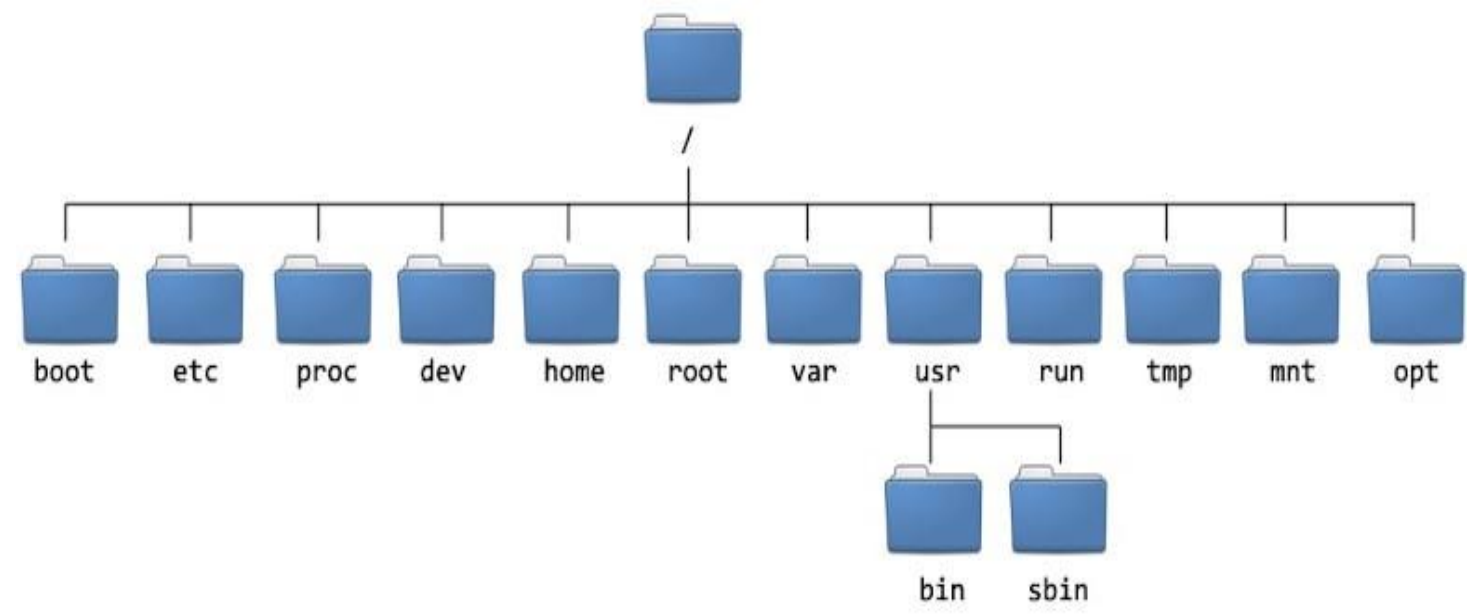

Fig.3. Hierarchy of the Linux file system

It should be noted that the servers and the "AzScienceNet" Data Center operate on the CentOS operating system. The data processing center plays an important role in solving complex problems that require large computational resources and memory at the institutes and organizations of ANAS.

The computing cluster Rocks is built in the "AzScienceNet" Data Center based on CentOS. This system includes 10 computers. The control panel of the system functions on the installed CentOS. The process of joining the computer system is performed automatically. At this time, CentOS are downloaded on the connected computer nodes. This downloading process is performed by the control machine.

\section{Conclusion}

The article explored available types of supercomputer architectures, including software and application of programming interfaces. A comparative analysis of software was implemented, and the dynamics of the distribution of operating systems over the last year in supercomputer technologies was investigated. In addition, CentOS software used in the scientific network "AzScienceNet" was analyzed. 


\section{References}

1. Voevodin V.V. Supercomputers: yesterday, today, and tomorrow. // Science and Life, 2000, No 5, pp.78-83.

2. Supercomputers (http://wikipedia.org/wiki/Supercomputer).

3. Tanenbaum E., Austin T. Computer architecture. SPB. Peter, 2013, 704 p.

4. Top 500 Supercomputers. (Http://top500.org/statistics/)

5. Architecture of computing systems. SMP and MPP architectures. (http://intuit.ru/studies/courses/45/45/lecture/1340)

6. Artem I.Yu. Cluster of high availability of software complex automation of data collection. // Software products and systems, 2013, No 4, pp.149-153.

7. Klimenko V.P. Komukhaev E.I. Supercomputers: Trends and technologies of the last two decades. // Mathematical machines and systems, 2006, No 3, pp.146-151.

8. Voevodin V.V., Voevodin Vl.V. Parallel computing, St. Petersburg: BHV, Petersburg, 2002, $608 \mathrm{p}$.

9. Korneyev V.D. Parallel programming of multicomputers in MPI. Novosibirsk: IPM and MG SB RAS, 2006, $126 \mathrm{p}$.

10. Tanenbaum E., Bos Kh. Modern Operational Systems. Piter, 2015.

11. Linux and parallelism (https://www.osp.ru/os/2003/05/183012/)

12. Windows HPC Server 2008, acquaintance and the first cluster. (http://hpcru.wordpress.com/2011/08/29/whpc2008-first-glance/)

13. Advantages and disadvantages of using CentOS. (http://realhosters.com/en/vps-oscentos.php) 